\title{
Polarized stimulated-emission depletion and dark-state lifetime at vacuum and cryogenic temperature conditions
}

\author{
Christiaan N. Hulleman $\odot$, Robert J. Moerland $\odot$, Sjoerd Stallinga $\odot,{ }^{*}$ and Bernd Rieger $\odot^{\dagger}$ \\ Department of Imaging Physics, Delft University of Technology, 2628 CJ Delft, The Netherlands
}

(Received 13 June 2021; revised 28 September 2021; accepted 25 October 2021; published 20 December 2021)

\begin{abstract}
With the growing popularity of cryogenic correlative light and electron microscopy, it is becoming increasingly important to bridge the resolution gap between these two modalities. At cryogenic temperatures, the photon yield of fluorophores is a few orders of magnitude higher than at room temperature, enabling localization precisions on the Ångström scale. The current challenge is to induce sparsity at cryogenic temperatures such that individual fluorescent molecules can be localized. In this paper, we demonstrate the progress of using polarized stimulated-emission depletion (STED) to induce sparsity at cryogenic temperatures and in vacuum. We generate linear polarization of arbitrary in-plane orientations to achieve polarized STED with a sparsity of 3.3:1. Furthermore, we have probed the dark-state lifetime of ATTO $647 \mathrm{~N}$ at cryogenic temperatures and in vacuum at room temperature. This dark state in vacuum is long-lived $(\tau=38 \mathrm{~ms})$ and could be the cause for reduced photostability of fluorophores under STED illumination in vacuum. The experiments were done on an in-house designed and built liquid nitrogen cryostat, enabling 30 hours of stable cryogenic fluorescence microscopy.
\end{abstract}

DOI: 10.1103/PhysRevA.104.063516

\section{INTRODUCTION}

The diffraction limited resolution in fluorescence microscopy can be circumvented with localization microscopy [1-3]. The localization precision primarily scales with wavelength $\lambda$, numerical aperture (NA) and number of captured photons $N$ as $\lambda /(\mathrm{NA} \sqrt{N})$ [1]. Performing localization microscopy at cryogenic temperatures has numerous advantages with this in mind. First, the sample can be fixed in a nearnative state without the need for chemical fixation, thereby eliminating fixation artifacts [4]. Second, the photobleaching rate of fluorophores is reduced at cryogenic temperatures [5]. Therefore, more photons can be extracted from each fluorophore, resulting in a better possible localization precision. The engineering challenge to maintain temperature gradients and minimize drift has been solved to some degree [6-11]. There is, however, still one major drawback to performing localization microscopy at cryogenic temperatures, which is the ability to induce sparsity. Sparsity is usually achieved by turning individual organic dyes on and off with either an active or stochastic process such that fluorophores can be localized individually.

Some success has been achieved with fluorescent proteins, which can switch on an off at cryogenic temperatures with a photon yield of $\sim 10000$ photons [12]. This is, however, a

\footnotetext{
*s.stallinga@tudelft.nl

†b.rieger@tudelft.nl
}

Published by the American Physical Society under the terms of the Creative Commons Attribution 4.0 International license. Further distribution of this work must maintain attribution to the author(s) and the published article's title, journal citation, and DOI. small fraction of the photon yield of organic dyes at cryogenic temperatures, where more than 1000000 photons are routinely achieved in sparse samples $[7,13,14]$. This hundredfold difference in photon yield translates to a ten times difference in localization precision [15]. An effective way to induce sparsity with organic dyes at cryogenic temperatures has not been reported, which implies that the high photon yield and associated localization precision cannot be fully utilized yet. The strength of cryogenic localization microscopy with organic dyes is, however, evident when using a limited and known number of dyes per biomolecule, and has been used to reveal the structure of proteins with Ångström resolution [16].

To induce sparsity at cryogenic temperatures, we have proposed the use of polarized stimulated-emission depletion (STED) in our earlier work [9]. This idea is based on the work of Hafi et al. where they used mutually orthogonal linearly polarized excitation and depletion beams to modulate the fluorescence to achieve super-resolution [17]. Their implementation, however, was later shown to owe its resolution enhancement primarily to the sparsity enhancing deconvolution and not the polarization modulation [18]. Their reported fluorescence modulation contrast before deconvolution was very low, presumably because both laser beams were rotated by a single $\lambda / 2$ retarder and any phase difference between polarizations induced in the other parts of the microscope were not accounted for. In our previous work, we solved this issue of low fluorescence modulation contrast through optimization of the polarization states for arbitrary polarization orientation angles [9]. Polarization STED with improved modulation contrast could be used to induce sparsity on samples with fixed dipole emitters, like those frozen in place at cryogenic temperatures. The induced sparsity could subsequently enable localizing single molecules to assemble a localization microscopy image. In this way, a genuine resolution 


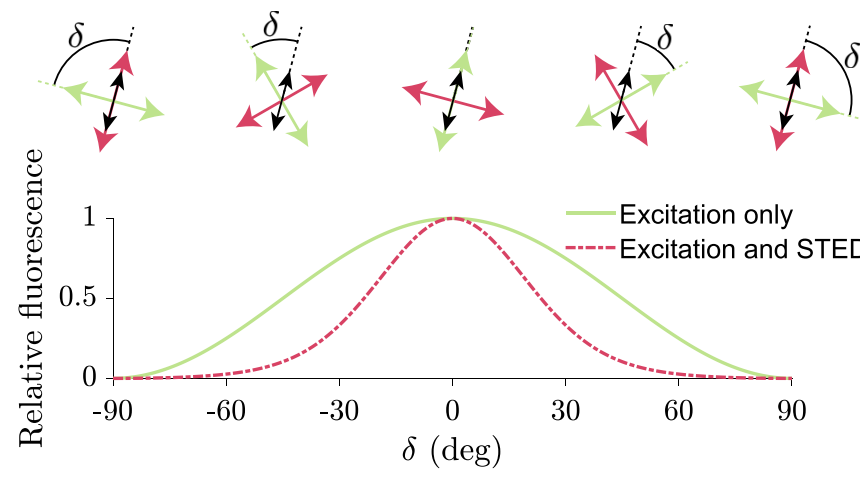

FIG. 1. Fluorophores with their fluorescent molecular dipole moment's in-plane projection illustrated in black can be excited by a linearly polarized excitation laser beam (solid light green). The relative emitted fluorescence scales with the inner product between them, proportional to $\cos ^{2} \delta$, where $\delta$ is the angle between the two in-plane orientations. With a linearly polarized STED laser beam (dash-dotted red), the relative fluorescence is suppressed for nonzero inner products between the dipole moment and the electric field vector of the STED beam. Arranging the in-plane excitation and depletion polarization perpendicular to each other, the angular subset of fluorescing molecules is reduced without reducing the fluorescence of fluorophores oriented in the desired direction.

enhancement could be achieved through localization microscopy at cryogenic temperatures, avoiding any deconvolution steps.

In this paper, we investigate the sparsity induced at cryogenic temperatures with polarized STED. The method is assessed by the degree of sparsity that can potentially be achieved and by the number of emitted photons per single molecule. It appears that longer lived triple states [19] limit the achievable photon yield. We have therefore probed this dark state for ATTO $647 \mathrm{~N}$ molecules and its recovery through ensemble-level measurements at cryogenic and room temperatures in vacuum. The experimental analysis makes use of pulsed excitation with variable off times, and includes measurements on the impact of a triplet-state quencher.

The outline of the paper is as follows. In Sec. II, we present the results of our experimental analysis of polarized STED on single molecules at cryogenic conditions. In Sec. III, we describe our experimental analysis of the buildup of and recovery from a long-lived dark state. The paper is concluded in Sec. IV with a discussion of the main findings of our paper. Several aspects of the experimental methods used (optical setup, cryostat, sample preparation) are described in the Appendices.

\section{POLARIZED STIMULATED-EMISSION DEPLETION AT CRYOGENIC TEMPERATURES}

The fluorescence of fixed dipoles can be modulated using in-plane linearly polarized light as illustrated in Fig. 1. If the fixed fluorescent dipole moment and excitation electric field are parallel, the excitation is most efficient and the relative fluorescence maximal. The relative fluorescence decreases as the angle $(\delta)$ between the fluorescent dipole moment and excitation electric field changes $\left(\sim \cos ^{2} \delta\right)$. The addition of an in-plane linearly polarized depletion beam with a perpendicular polarization to the excitation beam narrows the set of fixed emitters that have significant fluorescence [17]. The relative fluorescence is decreased as the depletion beam stimulates the emission of a photon identical to the incident depletion photon which is not detected on the camera as it is blocked by the fluorescence filters [20]. With the additional perpendicular depletion beam, the relative fluorescence scales with $\sim \cos ^{n} \delta$, where $n \geqslant 2$ increases with increasing STED laser power. With this angle narrowing, the set of orientations of effectively fluorescing dipoles is reduced with more STED laser power, realizing the sparsity needed for localization microscopy. For fluorescent dipole moments not aligned in plane (perpendicular to the optical axis), the maximal excitation efficiency will be reduced, however, molecules aligned along the desired in-plane projection $\left(\delta=0^{\circ}\right)$ will still not be depleted as the fluorescent dipole moment is perpendicular to the depletion polarization. To ensure that the fluorescent molecules oriented in the desired direction are not depleted, the STED beam needs to be linearly polarized. Any ellipticity in the polarization leads to a nonzero inner product between the depletion polarization and the fluorescent molecular dipole and, consequently, a nonzero chance of depletion.

We have built a setup for probing the fluorescence of single molecules at cryogenic conditions with polarized light, incorporating tight control of the polarization of the excitation and depletion beams. Details of the optical setup are described in Appendix A; the essentials of the design and operation of the cryostat can be found in Appendix B. The effectiveness of the polarization optimization can be seen from the excitation-only modulation of single ATTO $647 \mathrm{~N}$ molecules at cryogenic temperatures, irrespective of their orientation (dark green dash-dotted curves in Fig. 2). Using only a $\lambda / 2$ wave plate, it is not possible to generate diagonal linear polarization states at the sample due to the phase shift of the dichroic mirror. Instead, the polarization is elliptical and almost circular, as evident from the poor modulation of diagonally oriented molecules [solid blue curve, Fig. 2(b)].

We have used our setup to test the level of angle narrowing that can be achieved with polarized STED. To this end, we measured the fluorescence modulation of individual fluorophores with varying STED laser powers. The excitation and depletion efficiencies scale with the polar angle (angle with the optical axis) of individual fluorophores, therefore, a single stable molecule is selected to make a fair comparison of the angle narrowing as a function of STED laser power. At each STED laser power, two periods of polarization rotation are applied and the average normalized fluorescence intensities for selected STED laser powers are shown in Fig. 3(a). Here pulsed STED was utilized where $\sim 100$ ps excitation pulses are synchronized to $100-500$ ps STED pulses. The STED laser pulses are longer for higher incident laser powers due to self-phase modulation; consequently, the peak STED laser power does not scale linearly with incident power (Appendix C). The fluorescence intensity at different STED laser powers is fitted with the function $I=|a \cos (\delta+b)|^{n}+c$ to estimate the FWHM [Fig. 3(b)]. The uncertainty at $65 \mathrm{MW} / \mathrm{cm}^{2}$ is larger as the molecule was in a dark state during one of the periods. The smallest FWHM of $54^{\circ}$ occurred at $81 \mathrm{MW} / \mathrm{cm}^{2}$. With a FWHM of $54^{\circ}$, a sparsity of $180: 54$ or 3.3:1 can 

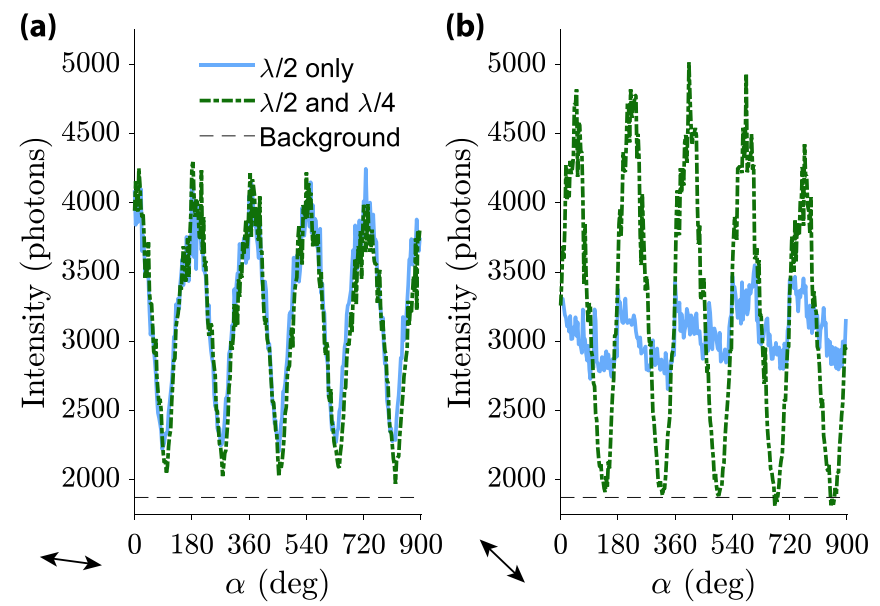

FIG. 2. Influence of polarization extinction ratio on the modulation contrast of single-molecule fluorescence. (a) Fluorescence modulation of an almost horizontally oriented single ATTO $647 \mathrm{~N}$ molecule $\left(\phi=7.7^{\circ} \pm 1.6^{\circ}\right)$. The blue solid curve only utilizes a $\lambda / 2$ wave plate to rotate the polarization; the dark green dash-dotted curve utilizes both a $\lambda / 2$ and $\lambda / 4$ wave plate to compensate for depolarization induced by the dichroic mirror. (b) Fluorescence modulation of an almost diagonally oriented molecule $\left(\phi=43.7^{\circ} \pm 1.1^{\circ}\right)$. The modulation contrast using only a $\lambda / 2$ wave plate is very low, as diagonal input polarization becomes elliptical after the dichroic mirror. The data is acquired with $2000 \mathrm{~ms}$ camera exposure and $1 \mathrm{~kW} / \mathrm{cm}^{2}$ excitation intensity.

be realized as $180^{\circ}$ covers all unique in-plane orientations. With continuous-wave polarized STED, this can be improved somewhat toward 6:1, which unfortunately is still far from hundreds or thousands to one required for dense biological samples [21].
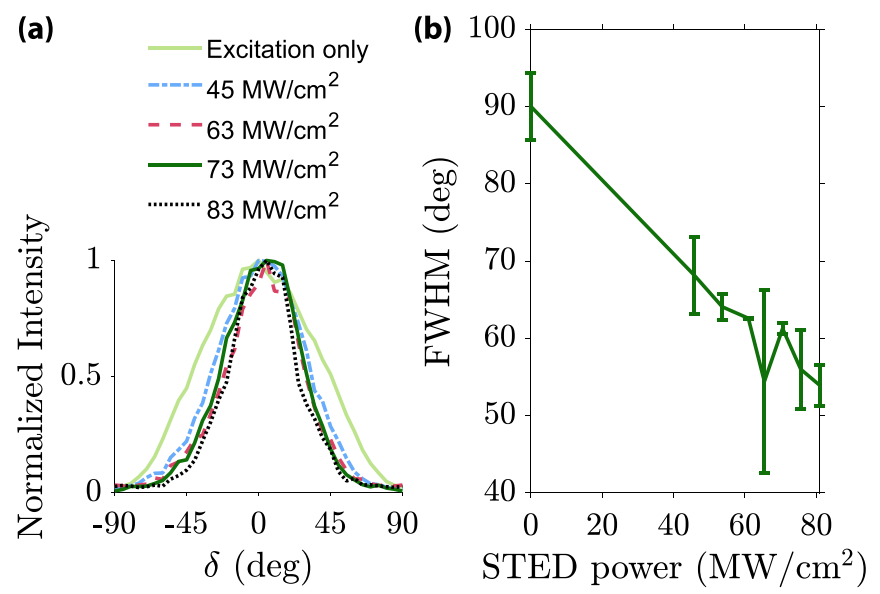

FIG. 3. FWHM reduction with increasing STED laser power. (a) Relative fluorescence intensity averaged over two periods of a single ATTO $647 \mathrm{~N}$ molecule illuminated with polarized STED with varying peak STED laser powers. The relative angle is the difference between the excitation polarization orientation angle and the in-plane orientation of the fluorescent molecular dipole moment $\left(\delta=\alpha_{\text {exc }}-\phi\right)$. (b) Angular FWHM at varying STED laser peak powers estimated from the fitted function. Error bars are \pm one standard deviation.
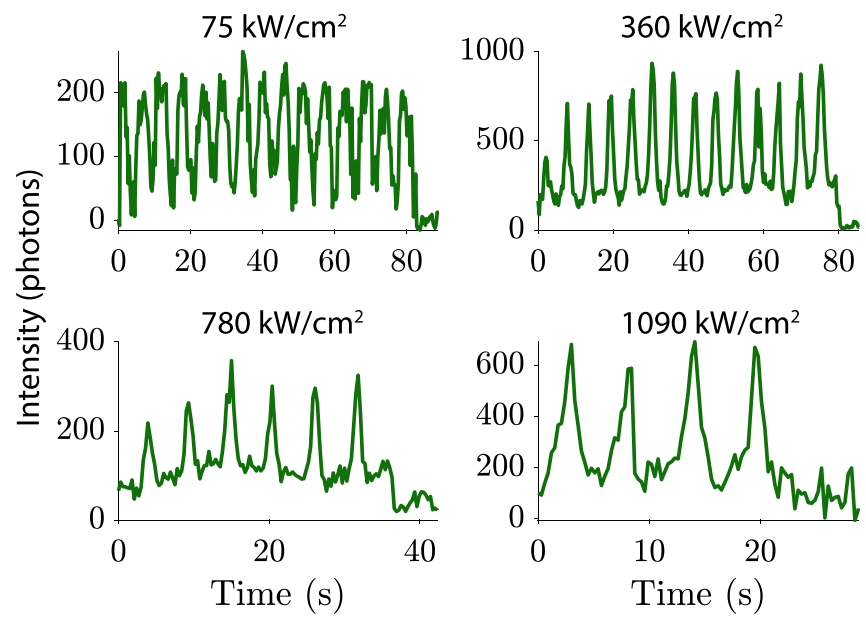

FIG. 4. Modulation of individual ATTO $647 \mathrm{~N}$ molecules at cryogenic temperatures from rotating linearly polarized STED and stationary circularly polarized excitation until bleaching. At higher STED laser powers, the survival time and photon yield is reduced. The data is acquired with $300 \mathrm{~ms}$ camera exposure and $1 \mathrm{~kW} / \mathrm{cm}^{2}$ excitation intensity.

In principle, the STED laser power could be increased a further fivefold with the available power from the laser, however, this leads to an unfeasible high intensity at the pulsestretching fiber facet. The temperature increase of the sample by addition of the STED laser is only $\Delta T \approx 9 \mathrm{~K}$ with $1 \mathrm{~W}$ average STED laser power, measured with PT1000 sensors in the copper sample holder $6 \mathrm{~mm}$ from the sample surface. This increased temperature is still well below the glass transition temperature of ice, and no dipole moment reorientation of the emitter is observed. The survivability of single molecules at cryogenic temperatures under polarized STED does decrease with increasing laser power. To illustrate this as well as the fact that the modulation is largely determined by the STED laser, we have imaged single ATTO 647N molecules at cryogenic temperatures where the excitation polarization is circularly polarized and only the polarization angle of the linearly polarized STED beam is rotated (Fig. 4). In this case, continuous-wave STED was used and the intensity is time averaged and therefore lower. The intensity per unit of time in Fig. 4 is similar to Fig. 2, but the exposure is $85 \%$ shorter to capture as many modulation periods as possible. The total number of photons captured from these individual molecules is 37675 photons at $75 \mathrm{~kW} / \mathrm{cm}^{2}, 98242$ photons at 360 $\mathrm{kW} / \mathrm{cm}^{2}, 16491$ photons at $780 \mathrm{~kW} / \mathrm{cm}^{2}$, and 21851 photons at $1090 \mathrm{~kW} / \mathrm{cm}^{2}$.

This amount of photons is 1-2 orders of magnitude below reported average photon yields of sparse single molecules at cryogenic temperatures [7,13-15]. One of the main bleaching pathways in STED microscopy is through the triplet state [22]. The STED process, however, also has a protective property as it rapidly depletes the singlet excited state, thereby reducing the transition rate to a triplet state in the first place [22]. Whether this protective property still plays an important roll at cryogenic temperatures and in vacuum is unclear as many of the underlying state-transition rates may be different. Typical cryogenic fluorescence microscopes have the sample 


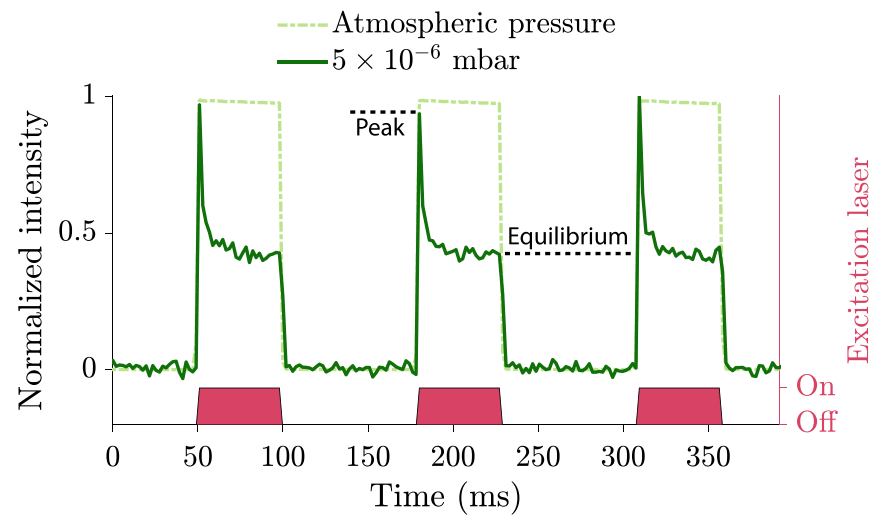

FIG. 5. Ensemble-level fluorescence emission of ATTO 647N under periodic illumination, with excitation laser on time of $50 \mathrm{~ms}$ and off time of $80 \mathrm{~ms}$. In vacuum (solid dark green), there is a rapid dark-state buildup, evident from the decrease in fluorescence intensity after onset of excitation illumination.

contained in either vacuum or an environment saturated with nitrogen gas, which could increase the triplet-state lifetime significantly. To determine if this could be a reason for the reduced photon yield with polarized STED, we investigate dark states that occur in vacuum and at cryogenic temperatures in the following section.

\section{DARK-STATE BUILDUP AND RECOVERY}

The triplet state plays a key role in the photophysics of single molecules [23]. Most typically, fluorophores have a triplet-state lifetime in the microsecond range [19,22,24]. In oxygen-deprived samples, for example, those with oxygen scavengers, the triplet-state lifetime is orders of magnitudes longer [25,26]. This is because oxygen plays a key role in quenching the fluorophore's triplet state through energy transfer from the fluorophore in a triplet state to oxygen in a triplet state [19]. If this prolonged triplet state is also present in vacuum and cryogenic systems, this should lead to observable dark states with dark-state lifetimes on the order of $1-100$ ms. For that reason, we have probed such dark states by intermittent excitation periods [27].

\section{A. Accumulation of fluorophores in a dark state in vacuum}

Periodically switching the excitation laser on and off allows the dynamics of the dark state of fluorophores to be probed on the ensemble level. The fluorescence intensity of ATTO $647 \mathrm{~N}$ molecules at atmospheric pressure follows the intensity of excitation when switching the laser on for $50 \mathrm{~ms}$ and then off for $80 \mathrm{~ms}$ (Fig. 5). There is no observable buildup into a dark state as the fluorescence intensity has a flat profile, which is to be expected as the triplet-state lifetime is on the order of a few $\mu$ s $[19,22,24]$. Pumping the same sample to a vacuum of $5 \times 10^{-6} \mathrm{mbar}$, a nonstable fluorescence intensity is observed under periodic illumination as a dark state builds up. After the onset of illumination, there is a sharp exponential decrease in fluorescence intensity that reaches an equilibrium level. Fitting a biexponential function to the buildup time reveals a characteristic timescale of $2.2 \pm 0.5 \mathrm{~ms}$. The dark-

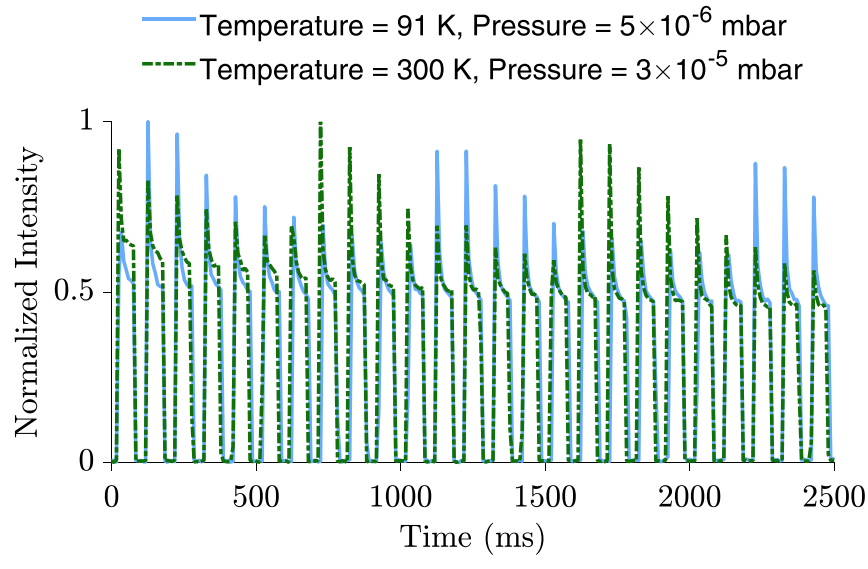

FIG. 6. Fluorescence modulation and dark-state buildup of ATTO $647 \mathrm{~N}$ in vacuum at room temperature (dash-dotted dark green) and cryogenic temperatures (solid blue). The excitation laser is modulated with $50 \mathrm{~ms}$ on time and $50 \mathrm{~ms}$ off time.

state buildup time could actually be faster as this measured value is on the same timescale as the frame time $(1.97 \mathrm{~ms})$. Not illuminating the sample for a sufficient amount of time allows the fluorophores to recover to the ground state. Thereafter, a peak in the fluorescence intensity will be observed when the excitation laser is turned on again.

The accumulation of this dark state is present at both room and cryogenic temperatures in vacuum (Fig. 6). From this figure, it can be seen that dark-state buildup and recovery times are similar, as is the ratio between the peak level and equilibrium level. There is also a clear problem with aliasing, as seen from the distinct time-varying peak intensity. This stems from the fact that the dark-state buildup is fast compared to the camera frame rate and that no photons are collected during frame readouts. The data in Fig. 6 is acquired with the EMCCD camera using $5 \mathrm{~ms}$ exposure and a frame rate of $120 \mathrm{fps}$. To reduce the frame readout time, an sCMOS camera was used for the following experiments along with Fig. 5, as this enables the use of $1 \mathrm{~ms}$ exposure and $508 \mathrm{fps}$ frame rate. As the difference between the dynamics of the dark state at cryogenic and room temperature is so small, the following experiments were performed at room temperature.

\section{B. Probing dark-state recovery}

To probe the dark-state recovery time, the off time between excitation pulses is varied. Longer off times allow more fluorophores to recover back to the ground state and, consequently, a higher peak fluorescence signal at the onset of the next excitation pulse. The influence of varying the off time can be seen from the time traces in Fig. 7 with 10 ms, 20 $\mathrm{ms}$, and $30 \mathrm{~ms}$ off times. By comparing the ratio of peak level to equilibrium level (peak divided by equilibrium) for varying off times, the dark-state lifetime can be measured.

To reduce the influence of aliasing each off time is measured for 40 periods and the highest five peak values are analyzed. The peak-equilibrium ratio shown in Fig. 8 varies smoothly as a function of the off-time and the standard deviation of the five analyzed ratios is small $(\widetilde{\sigma}=0.013)$. The change of the peak-equilibrium ratio as a function of off time 


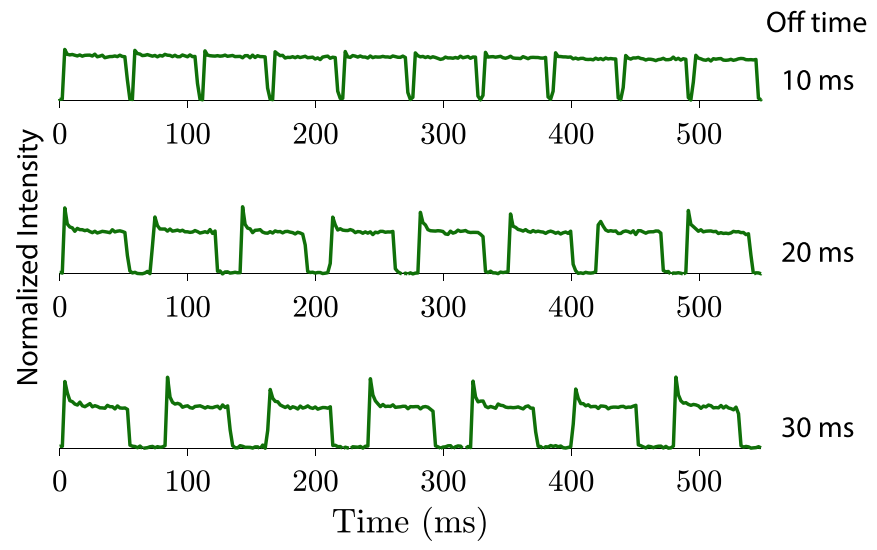

FIG. 7. Impact of the off time of excitation illumination on the recovery from the dark state. The amount of dark-state recovery scales with the off-time duration, as seen from the increasing peaks at the onset of illumination.

is well fitted by a double exponential function $c-a e^{-t_{\text {off }} / \tau_{1}}-$ $b e^{-t_{\text {off }} / \tau_{2}}$. For ensemble-level ATTO $647 \mathrm{~N}$, the recovery times at $4 \times 10^{-5} \mathrm{mbar}$ are $\tau_{1}=38 \mathrm{~ms}$ and $\tau_{2}=132 \mathrm{~ms}$. The found $\tau_{1}$ dark-state lifetime is similar to the triplet-state lifetime found in oxygen depleted aqueous solutions ( $28 \pm 7 \mathrm{~ms}$ [25] and $29 \pm 5 \mathrm{~ms}$ [26]). Analyzing more measurements lowers the peak-equilibrium ratio but has almost no influence on the calculated dark-state recovery time $\tau_{1}$.

\section{Dark-state lifetime reduction with Trolox}

In an attempt to reduce the dark-state lifetime in vacuum, we investigated the addition of Trolox. Trolox is known to function as an antiblinking and antibleaching agent by quenching the triplet state [28]. Comparing an ensemble level of spin-coated ATTO $647 \mathrm{~N}$ with and without $90 \mathrm{mM}$ of Trolox, the dark-state lifetime is reduced from $\tau_{1}=38 \mathrm{~ms}$ to $\tau_{1}=33 \mathrm{~ms}$, which can also be seen from the faster increase in the peak-equilibrium ratio in Fig. 9.

Instead of spin coating the $90 \mathrm{mM}$ concentration of Trolox, which creates a flat and essentially 2D layer, it is also possible

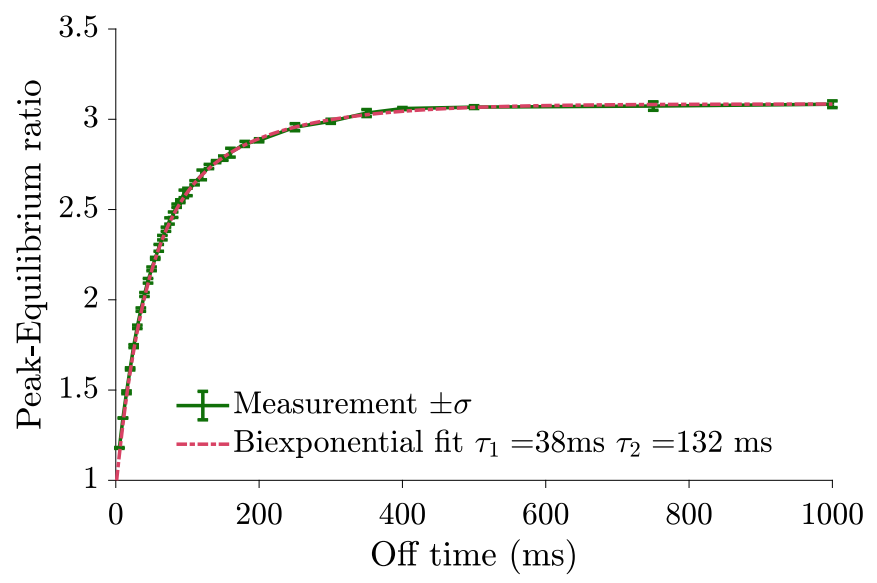

FIG. 8. The measured peak-equilibrium ratio as a function of off time (solid dark green), and fit with a biexponential function (red dash-dotted) $(\mathrm{RMSE}=0.006)$.

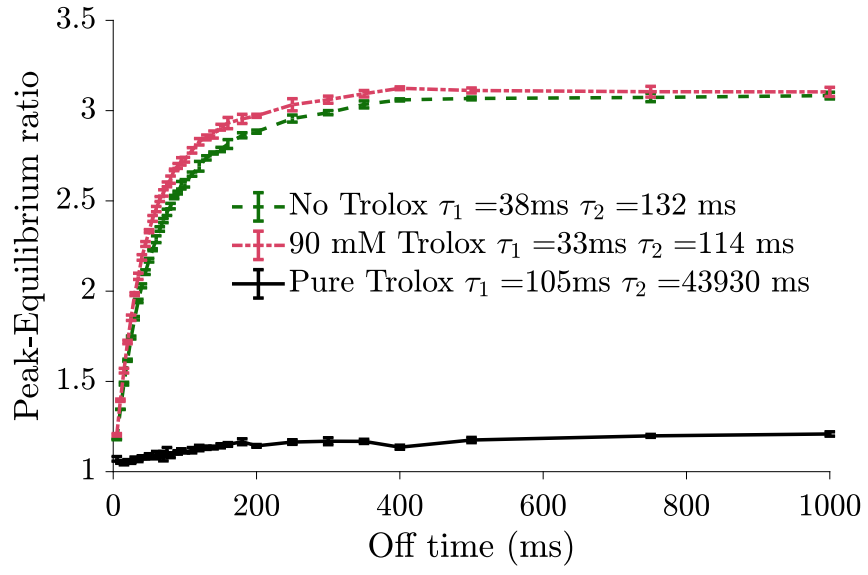

FIG. 9. Rapid dark-state recovery with the addition of Trolox. 90 $\mathrm{mM}$ of Trolox (red dash-dotted) in a thin layer of single molecules slightly reduces the dark-state lifetime. In a thick layer of pure Trolox (solid black), the dark-state buildup is largely eliminated

to create a sample by letting the dye and Trolox solution evaporate. In this way, a solid droplet of higher concentration Trolox is realized which we termed pure Trolox. At this high concentration of Trolox, the peak-equilibrium curve becomes flat around a value of 1 . This is because there is almost no observable buildup of the triplet state and the time traces look similar to the case of atmospheric pressure in Fig. 5. The initial dark-state lifetime around $\sim 30 \mathrm{~ms}$ of spincoated ensemble-level ATTO $647 \mathrm{~N}$ has disappeared, instead the slower decay time $\tau_{1}=105 \mathrm{~ms}$ is still present, which is comparable to the $\tau_{2} \approx 120 \mathrm{~ms}$ value from the spin-coated samples.

To mimic a thin biological sample, we have embedded ATTO $647 \mathrm{~N}$ and Trolox in a spin-coated layer of polyvinyl alcohol (PVA). This reduces the average distance between fluorophores and Trolox, as the molecules are not only spread in a single 2D layer but are distributed in 3D. In this thicker layer, a reduction in the peak-equilibrium ratio can be achieved at lower Trolox concentrations. Figure $10 \mathrm{com}$ pares the peak-equilibrium ratio of PVA samples with Trolox (dashed curve) and without Trolox (solid curve) at various pressures. At each pressure, the sample with Trolox has a significantly lower buildup of the dark state and therefore a lower peak-equilibrium ratio. The difference between atmospheric pressure and $\sim 2$ mbar is large for both the sample with and without Trolox. At atmospheric pressure, there appears to be an initial peak in the peak-equilibrium ratio which then reduces again and does not match the expected exponential behavior. This could possibly be caused by molecules diffusing into the PVA sample. Interestingly, further reducing the pressure from $\sim 2$ mbar to $\leqslant 10^{-5}$ mbar makes a relatively small difference in the peak-equilibrium ratio.

\section{CONCLUSIONS}

Polarized STED can be used to narrow the set of fixed fluorescing molecules at cryogenic temperatures. Unfortunately, the FWHM achieved with significant STED powers does not induce enough sparsity to perform localization microscopy on 


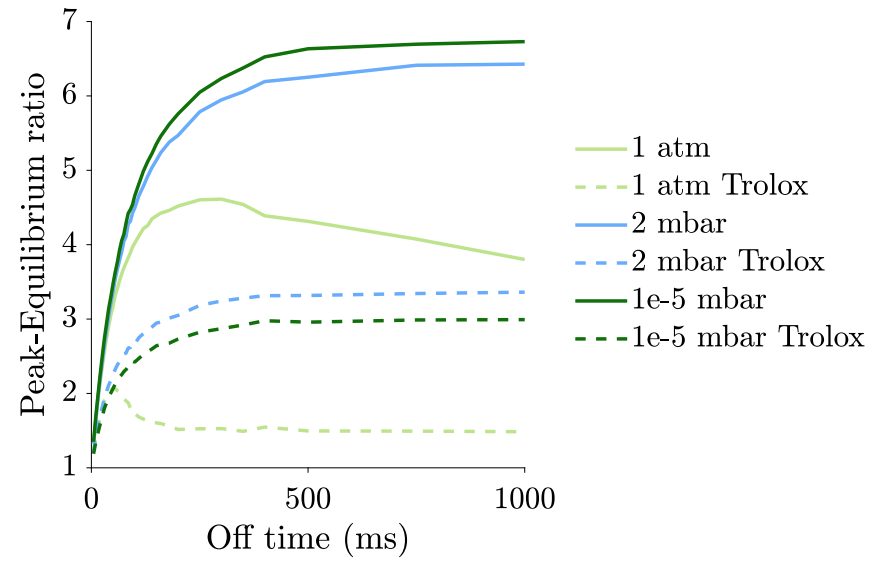

FIG. 10. Peak-equilibrium ratio of a $100 \mathrm{nM}$ concentration of ATTO 647N embedded in PVA with $4 \mathrm{mM}$ of Trolox (dashed lines) and without (solid lines) at varying pressures. The standard deviation of the measurements is comparable to those in Figs. 8 and 9 and error bars are left out for clarity.

dense biological samples. The level of sparsity achieved could be improved by varying the polarization states also toward the optical axis instead of only rotating the mutually perpendicular polarization states around the optical axis. This would approximately square the amount of sparsity but would be excessively complicated to realize in a low NA cryogenic microscope. An additional $27 \%$ better FWHM could be achieved using two-photon excitation as the basic excitation dependence is enhanced quadratically yielding $\operatorname{a~} \cos ^{4} \delta$ dependence [29], before inclusion of additional polarized STED. Next to the limited improvement in sparsity, the use of STED also has a negative impact on the photon yield of fluorophores at cryogenic temperatures. With the STED process, fluorophores are still going through the photocycle while they are in a virtual off state that is not imaged on the camera, thereby increasing the chance of photobleaching.

In our cryostat, the sample is contained in vacuum which causes a prolonged dark-state lifetime. The dark-state lifetime of ATTO $647 \mathrm{~N}$ in vacuum was found to be $\tau=38 \mathrm{~ms}$. Unfortunately, this dark-state lifetime is not significantly longer than the typical fluorescence on-state lifetime. If this was the case, it could be utilized to directly induce sparsity in samples in vacuum and at cryogenic temperatures. This prolonged dark-state lifetime, a few orders of magnitude longer than in ambient conditions, increases the chances of irreversible photobleaching of the fluorophores.

The average dark-state lifetime in vacuum can be decreased by the addition of Trolox. For a significant decrease of the dark-state lifetime, a very high concentration of Trolox needs to be added to fixed and nondiffusing samples. A standard sample in a liquid buffer can suffice with a lower concentration of Trolox as these molecules diffuse. This increases the chance of a Trolox molecule being in the proximity of a fluorophore in the triplet state. For fixed and frozen samples, it would be ideal to constrain stabilizing molecules like Trolox to the proximity of the fluorophore. A conjugate of covalently bound NPA (a photostabilizer) and ATTO 647N that stabilizes fluorescence emission and significantly reduces the triplet-state lifetime [26] can be very useful to further increase the photon yield of frozen fluorophores at cryogenic temperatures. With such a conjugation of dye and stabilizing molecules, the fluorescence emission is more robust without the need for excess amounts of chemicals in the imaging buffer.

Instead of localizing molecules, it is possible to use fluctuation-based algorithms like SOFI [10,30] in combination with the additional modulation from polarized STED, though this will not fully utilize the high photon yield available at cryogenic temperatures. Another potential approach would be an expansion along the lines of cryogenic optical localization in 3D (COLD) [16], where a small known number of fluorophores are only localized when it is certain from the fluorescence time trace that a single molecule is fluorescing during that frame. It should be possible to make an accurate estimation of a larger though still limited amount of molecules present in each time frame until all fluorophores are bleached, by utilizing the slow bleaching and high photon count at cryogenic temperatures along with polarization modulation. This information can then be combined with a multi-emitter fitting procedure [31,32]. This eliminates the typical problem of multi-emitter fitting where the number of emitters is unknown. The algorithm would then work in reverse chronological order starting with the frames with only one emitter per diffraction limited spot and sequentially fit frames with more emitters utilizing the previous localizations and varying contributions depending on the polarized modulation. Similar to using polarized excitation or detection to separate and assign emitted photons when it is known, there are two individual fluorophores within a diffraction limited spot [33], but expanded to determine the number of emitters from the data and combined with a complex fitting procedure. Such a multi-emitter and multiframe fitting procedure would be computationally intensive but beneficial to extract more information from the long imaging times of fixed dipole emitters at cryogenic temperatures.

\section{ACKNOWLEDGMENTS}

We thank Harald Homulle for loaning a GHz oscilloscope, Raymond Vermeulen for high-frequency cables, Ruud van Tol for designing the custom cryostat, Ron Hoogerheide for machining mechanical components, and Joost de Jong for preliminary pulse synchronization. This work was supported by the European Research Council (ERC) Grant No. 648580.

\section{APPENDIX A: OPTICAL SETUP}

The essential parts of the optical system as illustrated in Fig. 11 are the polarization control, pulse stretching of the depletion beam, and synchronization with the excitation pulses. The depletion beam path illustrated in red comes from a femtosecond tunable Ti:Sapphire laser (Mai Tai HP, SpectraPhysics) that goes through an optical isolator (IO-5780-HP, Thorlabs) to prevent back reflections into the laser cavity. A beam sampler (10B20-01NC.2, Newport) reflects $\sim 3 \%$ to a photodetector (DET025A/M, Thorlabs) to synchronize the excitation pulses. The depletion beam power is modulated by a $\lambda / 2$ wave plate (10RP52-2, Newport) and 


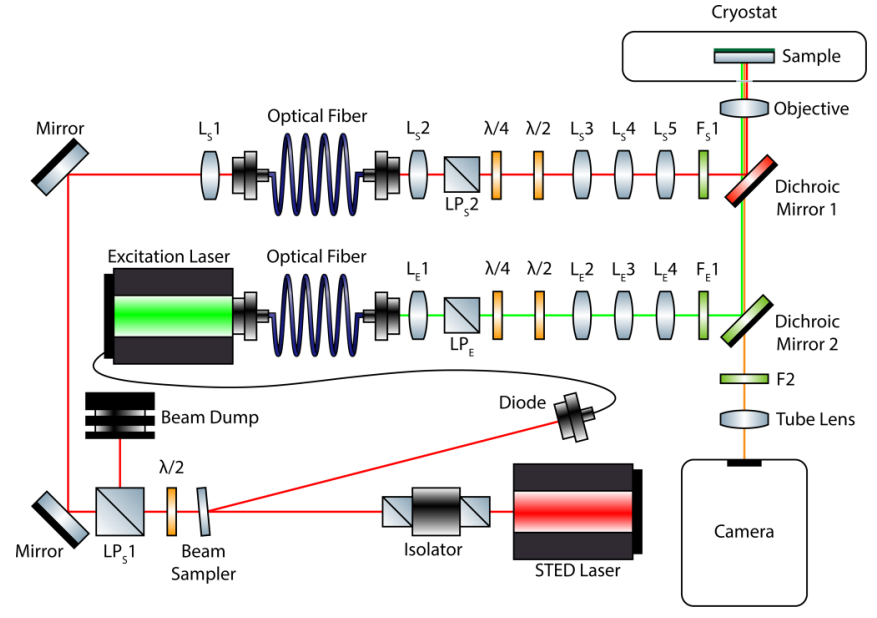

FIG. 11. Optical setup for polarized STED at cryogenic temperatures. For details, see Appendix A.

Glan-laser polarizer LP 1 (10GL08AR.16, Newport) which is reflected into a beamdump (PL15, Newport). Components with a subscript $\mathrm{S}$ are in the STED beam-path. The beam is then focused by a aspheric lens (C430TME-B, Thorlabs) into a 50-m-long polarization maintaining fiber (PM630-HP, Thorlabs) mounted on an actively controlled stage (NanoMax 300, Thorlabs). The feedback for this stage comes from an additional beam sampler (BSF10-B, Thorlabs) and photodiode (FDS1010, Thorlabs) placed between $\mathrm{L}_{\mathrm{S}} 2$ and the $\lambda / 4$ wave plate. The beam is collimated by an aspheric lens $\mathrm{L}_{\mathrm{S}} 2$ (C280TMD-B, Thorlabs) and polarized by the linear polarizer LPS 2 (GL10-B, Thorlabs). The $\lambda / 4$ (AQWP05M-980, Thorlabs) and $\lambda / 2$ (AHWP05M-980, Thorlabs) wave plates are mounted in rotation stages (8MPR16-1, Standa). $\mathrm{L}_{\mathrm{S}} 3$ (LA1229-B, Thorlabs) and $\mathrm{L}_{S} 4$ (LC1715-B, Thorlabs) reduce the beam size and $\mathrm{L}_{S} 5$ (LA1708-B, Thorlabs) focuses on the back focal plane of the objective lens (CFI S Plan Fluor ELWD 60XC, Nikon). The depletion beam spectrum is filtered by $\mathrm{F}_{\mathrm{S}} 1$ (FF01-775/46, Semrock) and reflected off dichroic mirror 1 (a custom short-pass filter with an edge wavelength of $\sim 700 \mathrm{~nm}$ and reduced phase shift between $s$ and $p$-polarized light between 700-800 nm, Chroma).

Colored in green is the excitation beam path, with components denoted with a subscript $\mathrm{E}$, which originates from a fiber-coupled picosecond pulsed laser source (LDH-D-C-640, Picoquant). Timing of the pulses is synchronized from the beam pickoff from the depletion beam which is mounted on a rail (RLA300/M, Thorlabs), allowing for fine-tuning of the timing between the two pulses. For coarse timing adjustments a coaxial delay box (DB64, SRS) is used. The beam is collimated by an aspheric lens $\mathrm{L}_{\mathrm{E}} 1$ (A397TM-A, Thorlabs). After a linear polarizer $\mathrm{LP}_{\mathrm{E}}(\mathrm{GL} 10-\mathrm{A}$, Thorlabs) the polarization state is altered by a $\lambda / 4$ (AQWP05M-600, Thorlabs) and $\lambda / 2$ (AHWP05M-600, Thorlabs) wave plate mounted in rotation stages (8MPR16-1, Standa). The beam is demagnified by $\mathrm{L}_{\mathrm{E}} 2$ (LA1229-B, Thorlabs) and $\mathrm{L}_{\mathrm{E}} 3$ (LC1715-B, Thorlabs). $\mathrm{L}_{\mathrm{E}} 4$ (LA1461-B, Thorlabs) focuses on the back focal plane of the objective lens. The excitation beam is filtered by $\mathrm{F}_{\mathrm{E}} 1$ (FF01-637/7, Semrock) and reflected off dichroic mirror 2 (FF652-DI01, Semrock). The fluorescence indicated with an
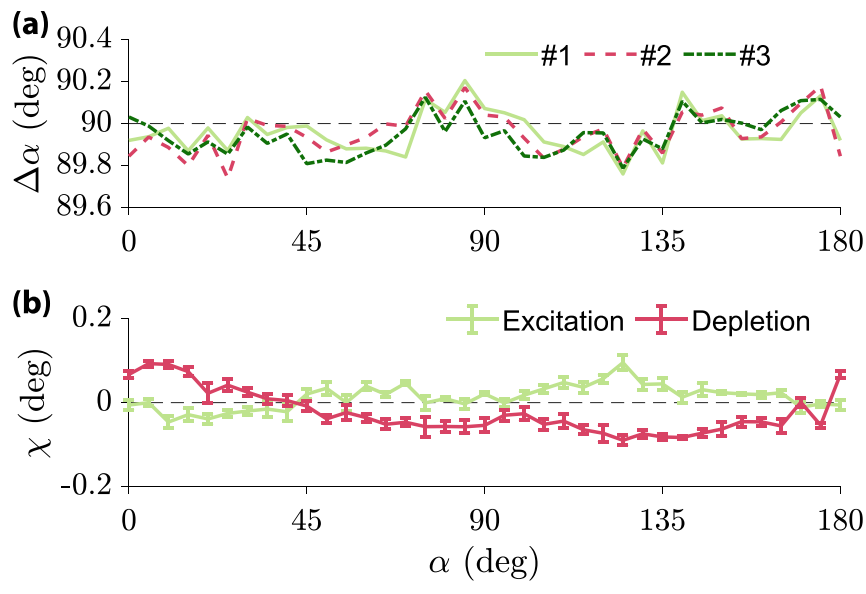

FIG. 12. Polarization orientation and ellipticity. (a) Relative angle between excitation and STED polarization $\Delta \alpha=\alpha_{\mathrm{exc}}-\alpha_{\mathrm{dep}}$ for three repeated measurements. (b) Ellipticity angle $(\chi)$ for the excitation (light green/light gray) and depletion (red/dark gray) polarization, error bars are \pm one standard deviation.

orange beam passes through the two dichroic mirrors and is filtered by F2, which consists of two (FF01-676/29, Semrock) filters and is then focused on the camera by a tube lens (ITL200, Thorlabs). For the polarized STED experiments, an EMCCD camera (iXon Ultra 888, Andor) was used and for probing the dark-state lifetime an sCMOS camera (Zyla 4.2 PLUS, Andor) was used.

The optical setup utilizes both a $\lambda / 4$ and $\lambda / 2$ wave plate for each beam path to rotate the polarization and compensate for any phase differences induced by the dichroic mirrors, following our earlier work [9]. The quality of polarization control is assessed with a dual rotating retarder polarimeter. Both the excitation beam and orthogonal depletion beam are corotated in $5^{\circ}$ steps. The angle difference between the two linearly polarized beams is $\Delta \alpha=\alpha_{\text {exc }}-\alpha_{\text {dep }}=89.96^{\circ} \pm 0.1^{\circ}$ with three repeated measurements shown in Fig. 12(a). The ellipticity angle for the excitation beam was $\chi=0.013^{\circ} \pm 0.03^{\circ}$ and for the depletion beam $\chi=-0.024^{\circ} \pm 0.05^{\circ}$ [Fig. 12(b)], giving insight into the level of polarization control that was achieved.

\section{APPENDIX B: CRYOSTAT}

The sample is contained in a cryostat that is a modified version of the one designed by Li et al. [7]. We doubled the liquid nitrogen capacity to three liters, allowing for a full day of imaging at cryogenic temperatures [9]. A drawback of their system is that for sample loading the heavy $(\sim 30 \mathrm{~kg})$ cryostat needs to be lifted. To alleviate this problem in our cryostat, the sample is loaded and imaged horizontally (Fig. 13) as opposed to the original vertical layouts $[7,9]$. This horizontal layout also eases the development of the microscope as optical components are easily accessible. Instead of translating the entire cryostat on large motorized stages, only the sample holder and thermal isolation bridge are translated with $x y$ piezo stages (Q-545.140, PI) inside the vacuum chamber. This allows for easy and accurate translation of the sample and the possibility for active drift correction. To minimize the heat transfer through radiation, the cold surfaces (heat conductor 


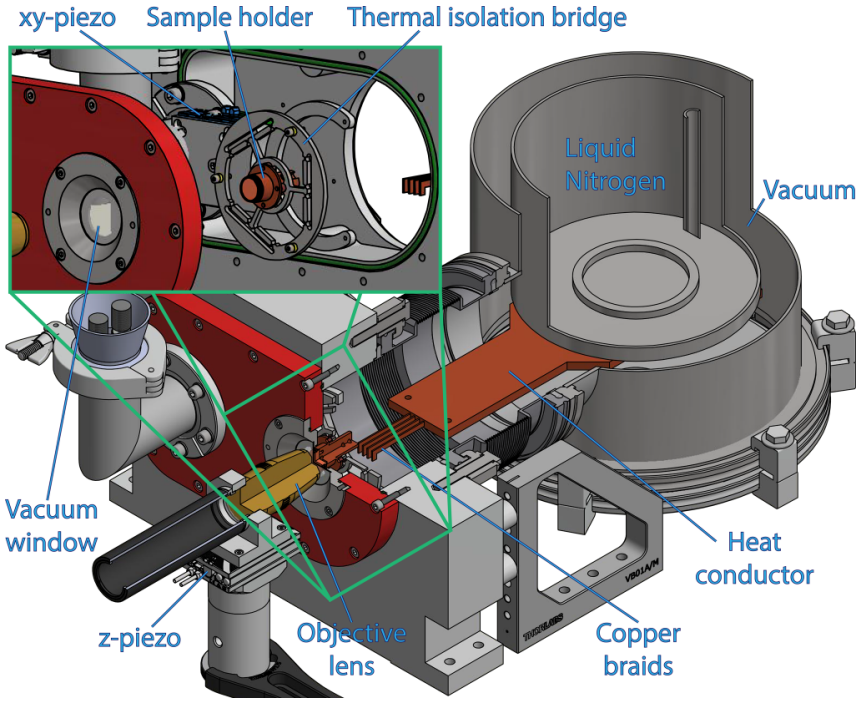

FIG. 13. Illustration of the liquid nitrogen cryostat with horizontal loading and $x y$ piezo stage in vacuum.

and liquid nitrogen tank) are encased with a ten-layer insulation foil (SCBnrc03, Cryoandmore). The connection from the sample holder to the thermal isolation bridge and to the piezo stage bracket is through polyetheretherketone (PEEK) spacers and screws, as PEEK has a low thermal conductivity and expansion coefficient. The objective mounted on a long-travel piezo stage (Q-545.240, PI) can be retracted and the vacuum window removed to exchange samples. A similar sample loading procedure to the one described by Li et al. [7] could potentially be used for frozen samples.

The vacuum chamber is pumped to a pressure $\leqslant 5 \times 10^{-5}$ mbar with a turbomolecular pump (TMU 065, Pfeiffer) and diaphragm roughing-pump (MVP 055-3, Pfeiffer), the pressure is measured with a full-range gauge (PKR 250, Pfeiffer). After pumping, the liquid nitrogen container is filled requiring $\sim 12$ liters to cool and completely fill the cryostat. Once the temperature is stabilized, after about an hour, the vacuum valve is closed and the pumps can be switched off as a vacuum of $\sim 1 \times 10^{-5}$ mbar is maintained by the large cold surface at liquid nitrogen temperature. With this cryostat, the sample can be kept at cryogenic temperatures for $30 \mathrm{~h}$ with a smooth pressure increase from $1.2 \times 10^{-5} \mathrm{mbar}$ to $8.3 \times 10^{-5} \mathrm{mbar}$. Over this $30 \mathrm{~h}$ period, a sample temperature of $T_{\mathrm{s}}=90.48 \pm$ $0.17 \mathrm{~K}$ can be realized with a very small average temperature slope of $\Delta T_{\mathrm{s}}=0.04 \mathrm{~K} / \mathrm{h}$ and maximally $\Delta T_{\mathrm{s}}=0.13 \mathrm{~K} / \mathrm{h}$ [Fig. 14(a)]. The thermal isolation bridge $T_{\mathrm{b}}=-17 \pm 1.3^{\circ} \mathrm{C}$ reduces the thermal energy flux from the piezo stage bracket $T_{\mathrm{p}}=12.3 \pm 1.2{ }^{\circ} \mathrm{C}$ toward the sample. The temperature of the copper heat conduction block $T_{\mathrm{c}}=81.63 \pm 0.02 \mathrm{~K}$ is higher than liquid nitrogen temperature $(77 \mathrm{~K})$ as the sensor is mounted closer to the copper braids. Temperatures are measured with PT1000 sensors and a four-channel data logger (PT-104, Picotech). Cooling of the sample can be done at a rate of $850 \mathrm{~K} / \mathrm{h}$ and the rate of temperature increase after all the liquid nitrogen is evaporated is only $18.5 \mathrm{~K} / \mathrm{h}$, which illustrates the thermal isolation quality.

With the addition of a weak cylindrical lens, astigmatic localization can be used to determine the 3D drift from beads (a)

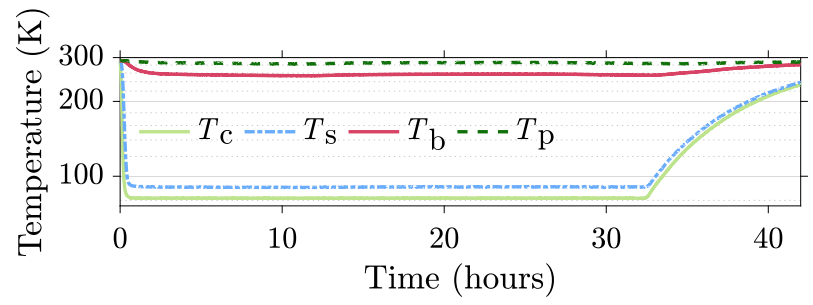

(b)

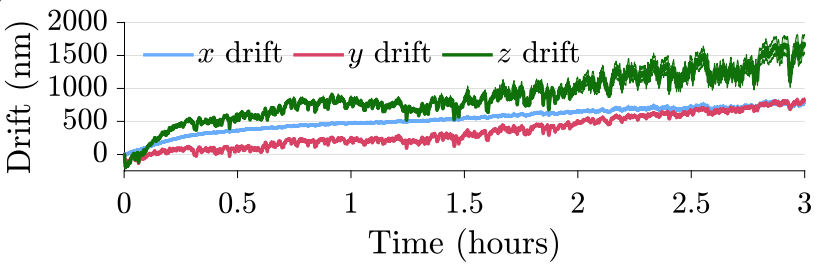

FIG. 14. Temperature and drift of the sample in the cryostat. (a) Temperature of the heat conductor $T_{\mathrm{c}}$ (bottom solid light green), sample holder $T_{\mathrm{s}}$ (dash-dotted light blue), thermal isolation bridge $T_{\mathrm{b}}$ (upper solid red), and piezo stage bracket $T_{\mathrm{p}}$ (dashed dark green) throughout an entire liquid nitrogen filled cycle. (b) Drift determined by $3 \mathrm{D}$ astigmatic localization of five fluorescent beads (shaded region represents \pm one standard deviation).

[34]. Here we used a 4 meter focal-length lens (4000 YO 25, Comar Optics) and a vectorial PSF model [35] to estimate the optical aberrations $\left(Z_{2}^{-2}=-133 m \lambda\right.$ and $\left.Z_{2}^{2}=55 m \lambda\right)$ from a $z$ stack and thereafter the $3 \mathrm{D}$ position of multiple beads. The $x y$ drift is only $\sim 800 \mathrm{~nm}$ over $3 \mathrm{~h}$ measured by localizing five fluorescent beads over 6320 frames [Fig. 14(b)]. The $z$ drift is slightly worse, up to $\sim 1600 \mathrm{~nm}$ over $3 \mathrm{~h}$. The majority of drift occurs during the first 20 minutes after onset of laser illumination. The drift is relatively smooth such that it can be corrected for in postprocessing or, alternatively, an active drift correction method could be implemented.

\section{APPENDIX C: SELF-PHASE MODULATION}

Pulsed STED requires well-synchronized and accurate timing of pulses for an effective depletion of the fluorescence. There is also an optimal depletion pulse length typically on the order of $100 \mathrm{ps}$, depending on the fluorophore and experimental parameters [36]. To attain these pulse lengths, the femtosecond laser pulses need to be stretched, here this is done by propagation through a polarization maintaining fiber. With the dispersion in the fiber, different wavelengths from the narrow spectrum of the depletion beam travel at different speeds and thus the pulse is stretched. At high instantaneous powers, however, nonlinear effects play a role where the local intensity changes the local refractive index. This is called self-phase modulation and leads to longer pulses [37]. Figure 15(a) shows the nonconstant pulse length as a function of the incident laser power.

These measurements are performed with a $12.5 \mathrm{GHz}$ diode (818-BB-45, Newport) and a $13 \mathrm{GHz}$ oscilloscope (WaveMaster $8 \mathrm{Zi}-\mathrm{B}$, Teledyne Lecroy). Each data point is the average of at least 1000 measurements. To maintain a good signal-to-noise ratio and avoid clipping, the signal after the pulse stretching fiber is measured after two additional linear polarizers (GL10-B, Thorlabs). The first eliminates the un- 

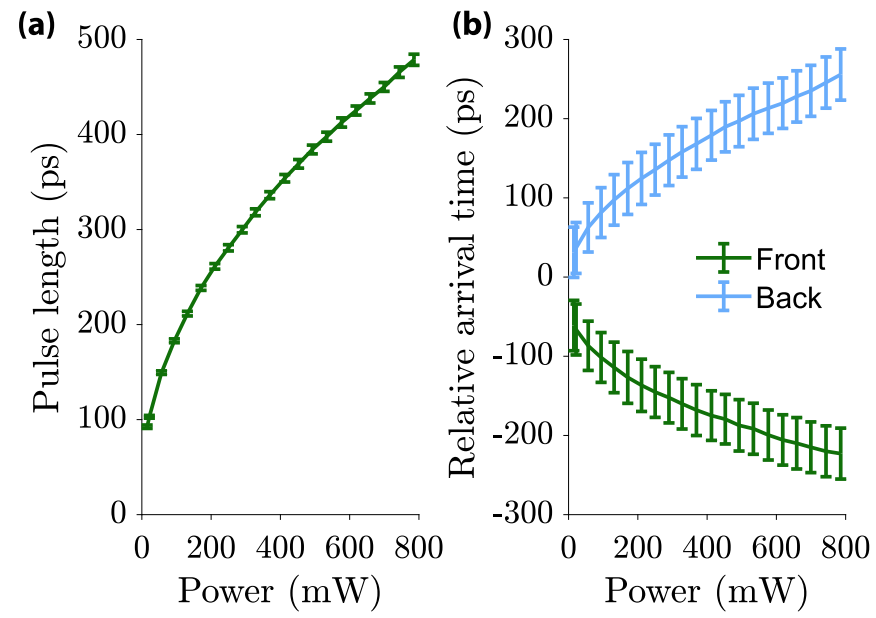

FIG. 15. Self-phase modulation in the pulse-stretching fiber at a wavelength of $780 \mathrm{~nm}$. (a) Pulse length (FWHM) as a function of incident laser power. (b) Relative arrival time of the beginning (dark green/dark gray) and end (blue/light gray) of the laser pulse as a function of laser power.

desired polarization and the second optimizes the intensity on the diode. The relative arrival times can be calculated by correlating the arrival times of the stretched pulse to the source pulse from the beam sampler and diode that are usually used to trigger the excitation pulses. Figure 15(b) shows that the onset of the stretched pulse arrives earlier as the power is increased and the tail of the pulse is delayed.

The increased pulse duration due to self-phase modulation comes along with spectral broadening [37]. Standard dichroic mirrors have a phase difference between $s$ - and $p$-polarized light that can vary rapidly as a function of wavelength. In this case the spectral broadening could lead to a significant increase in the polarization ellipticity angle. Fortunately, with the phase optimized dichroic mirror there is only $\sim 4 \%$ phase difference from $700 \mathrm{~nm}$ to $800 \mathrm{~nm}$. Therefore, the spectral broadening does not directly decrease the polarization extinction ratio, though it could decrease the depletion efficiency.

\section{APPENDIX D: SAMPLE PREPARATION AND IMAGE ANALYSIS}

The sparse single-molecule level ATTO 647N samples for excitation modulation and polarized STED are prepared by spin coating. ATTO 647N (ATTO-TEC) is diluted in Milli-Q (MQ) water to a concentration of $100 \mathrm{pM}$. $10 \mu \mathrm{L}$ of this solution is placed on a 12-mm-diameter quartz coverslip (amcoss) in the spin coater (SPIN150i, POLOS). The coverslips are precleaned by sonication for $1 \mathrm{~h}$ sequentially in ethanol (Honeywell), acetone (Honeywell), and ethanol again. As the quartz coverslips are heat resistant, they can also be recycled through an additional step by burning with a Bunsen burner [7]. The spin coater accelerates at a rate of $100 \mathrm{RPM} / \mathrm{s}$ to a rotation speed of $3000 \mathrm{RPM}$; after $3.5 \mathrm{~min}$ the solution is evaporated and the sample decelerates and can be mounted in the cryostat.

For the dark-state lifetime measurements, an ensemble level sample is made. From the sparse single-molecule samples, an estimate of the fluorophore density is made $\left(0.04 / \mu \mathrm{m}^{2}\right)$. The concentration of ATTO $647 \mathrm{~N}$ is then scaled up to $1 \mu \mathrm{M}$ to achieve a fluorohore density $\sim 400 / \mu \mathrm{m}^{2}$ such that each fluorophore is typically separated by $50 \mathrm{~nm}$. This is to avoid the influence of Förster resonance energy transfer, which becomes relevant at fluorophore separation distances $<10 \mathrm{~nm}$ [38].

A $100 \mathrm{mM}$ Trolox solution is made by combining $100 \mathrm{mg}$ of Trolox (6-hydroxy-2,5,7,8-tetramethylchromane2-carboxylic acid, Sigma-Aldrich), $430 \mu \mathrm{L}$ of methanol (Sigma-Aldrich), $345 \mu \mathrm{L} \mathrm{NaOH}$ (Sigma-Aldrich) and $3.2 \mathrm{~mL}$ of MQ water [39]. Thereafter, $90 \mu \mathrm{L}$ of this solution is mixed with $10 \mu \mathrm{L}$ of a $10 \mu \mathrm{M}$ solution of ATTO $647 \mathrm{~N}$, resulting in a final solution with $90 \mathrm{mM}$ Trolox and $1 \mu \mathrm{M}$ of ATTO $647 \mathrm{~N}$. For the two different samples, this is either applied to a quartz coverslip with the same spin-coating protocol mentioned above or by letting a $20 \mu \mathrm{L}$ droplet evaporate directly on the coverslip.

For ensemble-level ATTO $647 \mathrm{~N}$ in PVA, 1 gram of PVA (Mowiol 4-88, Sigma-Aldrich) is dissolved in 200-mL MQ water to create a $0.5 \mathrm{w} \%$ PVA solution. Thereafter, $475 \mu \mathrm{L}$ of the PVA solution is mixed with $20 \mu \mathrm{L}$ of the $100 \mathrm{mM}$ Trolox solution and $5 \mu \mathrm{L}$ of a $10 \mu \mathrm{M}$ ATTO $647 \mathrm{~N}$ solution. This is then applied to a quartz coverslip with the same spin-coating protocol mentioned above.

For localization microscopy, an accurate PSF model should be used that accounts for the dipole emission of fixed fluorophores. To analyze the modulation of individual fluorescent molecules, it is sufficient to simply count the amount of photons in a region of interest $(7 \times 7)$ around the fluorophore [40]. A gain calibration (gain $0.017 e^{-} /$ADU and offset 500.7 ADU with an EM gain setting of 100) is performed to convert analog-to-digital units to photon counts [41]. For the ensemble level measurements, the fluorescence intensity is summed from the inner region of the excitation spot.
[1] E. Betzig, G. H. Patterson, R. Sougrat, O. W. Lindwasser, S. Olenych, J. S. Bonifacino, M. W. Davidson, J. LippincottSchwartz, and H. F. Hess, Imaging intracellular fluorescent proteins at nanometer resolution, Science 313, 1642 (2006).

[2] S. T. Hess, T. P. Girirajan, and M. D. Mason, Ultra-high resolution imaging by fluorescence photoactivation localization microscopy, Biophys. J. 91, 4258 (2006).

[3] M. J. Rust, M. Bates, and X. Zhuang, Sub-diffractionlimit imaging by stochastic optical reconstruction microscopy (STORM), Nat. Methods 3, 793 (2006).
[4] M. Adrian, J. Dubochet, J. Lepault, and A. W. McDowall, Cryo-electron microscopy of viruses, Nature 308, 32 (1984).

[5] W. E. Moerner and M. Orrit, Illuminating single molecules in condensed matter, Science 283, 1670 (1999).

[6] R. Kaufmann, P. Schellenberger, E. Seiradake, I. M. Dobbie, E. Y. Jones, I. Davis, C. Hagen, and K. Grünewald, Superresolution microscopy using standard fluorescent proteins in intact cells under cryo-conditions, Nano Lett. 14, 4171 (2014). 
[7] W. Li, S. C. Stein, I. Gregor, and J. Enderlein, Ultrastable and versatile widefield cryo-fluorescence microscope for single-molecule localization with sub-nanometer accuracy, Opt. Express 23, 3770 (2015).

[8] R. Faoro, M. Bassu, Y. X. Mejia, T. Stephan, N. Dudani, C. Boeker, S. Jakobs, and T. P. Burg, Aberration-corrected cryoimmersion light microscopy, Proc. Nat. Acad. Sci. USA 115, 1204 (2018).

[9] C. N. Hulleman, M. Huisman, R. J. Moerland, D. Grünwald, S. Stallinga, and B. Rieger, Fluorescence polarization control for on-off switching of single molecules at cryogenic temperatures, Small Methods 2, 1700323 (2018).

[10] F. Moser, V. Prazák, V. Mordhorst, D. M. Andrade, L. A. Baker, C. Hagen, K. Grünewald, and R. Kaufmann, Cryo-SOFI enabling low-dose super-resolution correlative light and electron cryo-microscopy, Proc. Nat. Acad. Sci. USA 116, 4804 (2019).

[11] D. P. Hoffman, G. Shtengel, C. S. Xu, K. R. Campbell, M. Freeman, L. Wang, D. E. Milkie, H. A. Pasolli, N. Iyer, J. A. Bogovic, D. R. Stabley, A. Shirinifard, S. Pang, D. Peale, K. Schaefer, W. Pomp, C. L. Chang, J. Lippincott-Schwartz, T. Kirchhausen, D. J. Solecki et al., Correlative three-dimensional super-resolution and block-face electron microscopy of whole vitreously frozen cells, Science 367, 265 (2020).

[12] P. D. Dahlberg, A. M. Sartor, J. Wang, S. Saurabh, L. Shapiro, and W. E. Moerner, Identification of PAmKate as a red photoactivatable fluorescent protein for cryogenic super-resolution imaging, J. Am. Chem. Soc. 140, 12310 (2018).

[13] S. Weisenburger, B. Jing, A. Renn, and V. Sandoghdar, Cryogenic localization of single molecules with angstrom precision, Nanoimaging and Nanospectroscopy 8815, 88150D (2013).

[14] S. Weisenburger, B. Jing, D. Hänni, L. Reymond, B. Schuler, A. Renn, and V. Sandoghdar, Cryogenic colocalization microscopy for nanometer-distance measurements, ChemPhysChem 15, 763 (2014).

[15] C. N. Hulleman, W. Li, I. Gregor, B. Rieger, and J. Enderlein, Photon Yield Enhancement of Red Fluorophores at Cryogenic Temperatures, ChemPhysChem 19, 1774 (2018).

[16] S. Weisenburger, D. Boening, B. Schomburg, K. Giller, S. Becker, C. Griesinger, and V. Sandoghdar, Cryogenic optical localization provides 3D protein structure data with Angstrom resolution, Nat. Methods 14, 141 (2017).

[17] N. Hafi, M. Grunwald, L. S. van den Heuvel, T. Aspelmeier, J.-H. Chen, M. Zagrebelsky, O. M. Schütte, C. Steinem, M. Korte, A. Munk, and P. J. Walla, Fluorescence nanoscopy by polarization modulation and polarization angle narrowing., Nat. Methods 11, 579 (2014).

[18] L. Frahm and J. Keller, Polarization modulation adds little additional information to super-resolution fluorescence microscopy, Nat. Methods 13, 7 (2016).

[19] S. Van De Linde, I. Krstić, T. Prisner, S. Doose, M. Heilemann, and M. Sauer, Photoinduced formation of reversible dye radicals and their impact on super-resolution imaging, Photochem. Photobiol. Sci. 10, 499 (2011).

[20] S. W. Hell and J. Wichmann, Breaking the diffraction resolution limit by stimulated emission: Stimulated-emission-depletion fluorescence microscopy, Opt. Lett. 19, 780 (1994).

[21] S. van de Linde, A. Löschberger, T. Klein, M. Heidbreder, S. Wolter, M. Heilemann, and M. Sauer, Direct stochastic optical reconstruction microscopy with standard fluorescent probes, Nat. Protoc. 6, 991 (2011)

[22] G. Donnert, J. Keller, R. Medda, M. A. Andrei, S. O. Rizzoli, R. Lührmann, R. Jahn, C. Eggeling, and S. W. Hell, Macromolecular-scale resolution in biological fluorescence microscopy, Proc. Nat. Acad. Sci. USA 103, 11440 (2006).

[23] B. Kozankiewicz and M. Orrit, Single-molecule photophysics, from cryogenic to ambient conditions, Chem. Soc. Rev. 43, 1029 (2014).

[24] J. Widengren, U. Mets, and R. Rigler, Fluorescence correlation spectroscopy of triplet states in solution: A theoretical and experimental study, J. Phys. Chem. 99, 13368 (1995).

[25] C. Steinhauer, C. Forthmann, J. Vogelsang, and P. Tinnefeld, Superresolution microscopy on the basis of engineered dark states, J. Am. Chem. Soc. 130, 16840 (2008).

[26] J. H. M. van der Velde, J. Oelerich, J. Huang, J. H. Smit, A. Aminian Jazi, S. Galiani, K. Kolmakov, G. Gouridis, C. Eggeling, A. Herrmann, G. Roelfes, and T. Cordes, A simple and versatile design concept for fluorophore derivatives with intramolecular photostabilization, Nat. Commun. 7, 10144 (2016).

[27] A. Giske, CryoSTED microscopy-A new spectroscopic approach for improving the resolution of STED microscopy using low temperature, Ph.D. thesis, Ruperto-Carola University Heidelberg, 2007.

[28] T. Cordes, J. Vogelsang, and P. Tinnefeld, On the mechanism of Trolox as antiblinking and antibleaching reagent, J. Am. Chem. Soc. 131, 5018 (2009).

[29] J. R. Lakowicz, I. Gryczynski, J. Kuśba, and E. Danielsen, Two photon-induced fluorescence intensity and anisotropy decays of diphenylhexatriene in solvents and lipid bilayers, J. Fluoresc. 2, 247 (1992).

[30] T. Dertinger, R. Colyer, G. Iyer, S. Weiss, and J. Enderlein, Fast, background-free, 3D super-resolution optical fluctuation imaging (SOFI), Proc. Nat. Acad. Sci. USA 106, 22287 (2009).

[31] S. J. Holden, S. Uphoff, and A. N. Kapanidis, DAOSTORM: An algorithm for high-density super-resolution microscopy, Nat. Methods 8, 279 (2011).

[32] F. Huang, S. L. Schwartz, J. M. Byars, and K. A. Lidke, Simultaneous multiple-emitter fitting for single molecule superresolution imaging, Biomed. Opt. Express 2, 1377 (2011).

[33] D. Böning, F. F. Wieser, and V. Sandoghdar, Polarizationencoded colocalization microscopy at cryogenic temperatures, ACS Photonics 8, 194 (2021).

[34] B. Huang, W. Wang, M. Bates, and X. Zhuang, Three-dimensional super-resolution reconstruction microscopy, Science 319, 810 (2008).

[35] M. Siemons, C. N. Hulleman, R. Ø. Thorsen, C. S. Smith, and S. Stallinga, High precision wavefront control in point spread function engineering for single emitter localization, Opt. Express 26, 8397 (2018).

[36] M. Leutenegger, C. Eggeling, and S. W. Hell, Analytical description of STED microscopy performance, Opt. Express 18, 26417 (2010)

[37] E. Auksorius, Multidimensional fluorescence imaging and super-resolution exploiting ultrafast laser and supercontinuum technology, Ph.D. thesis, Imperial College London, 2008.

[38] I. H. Stein, V. Schüller, P. Böhm, P. Tinnefeld, and T. Liedl, Single-molecule FRET ruler based on rigid DNA origami blocks, ChemPhysChem 12, 689 (2011). 
[39] J. Schnitzbauer, M. T. Strauss, T. Schlichthaerle, F. Schueder, and R. Jungmann, Super-resolution microscopy with DNA-PAINT, Nat. Protoc. 12, 1198 (2017).

[40] R. Ø. Thorsen, C. N. Hulleman, M. Hammer, D. Grünwald, S. Stallinga, and B. Rieger, Impact of optical aberrations on axial position determination by photometry, Nat. Methods 15, 989 (2018).

[41] L. J. van Vliet, D. Sudar, and I. T. Young, Digital fluorescence imaging using cooled charge-coupled device array cameras, in Cell Biology, edited by J. E. Celis (Academic Press, New York, 1998), Second Edition, Vol. III, pp. 109-120. 